\title{
Die öffentliche Förderung des Breitbandausbaus, eine kritische Würdigung
}

\author{
Gordon J. Klein
}

Online publiziert: 20. November 2020

(C) Der/die Autor(en) 2020

Zusammenfassung Die Digitalisierung als ein Querschnittsthema betrifft verschiedenste Ebenen des wirtschaftlichen und gesellschaftlichen Lebens. Es ist somit wenig überraschend, dass Politik diesen Prozess gestalten möchte. Dabei werden verschiedene Akzente durch Förderprogramme gesetzt, von denen einige beispielhaft dargestellt werden. Im besonderen Fokus dieser Arbeit steht das Bundesbreitbandförderprogramm. Hier wird zunächst die Lage bezüglich des Ausbaus aufgezeigt, eine Übersicht über heterogene Fördermaßnahmen dargelegt und dann ein normativer Eingriffsbedarf ermittelt. Es folgt eine kritische Würdigung der aktuellen Förderung, bei der dargestellt wird, dass diese sehr weitgehend ist und dabei wahrscheinlich auch dort fördert, wo es auch ohne Förderung zu einem Ausbau gekommen wäre.

Schlüsselwörter Breitbandausbau · Förderung · Qualitative Evaluation

G. J. Klein $(\bowtie)$

CAWM - Centrum für angewandte Wirtschaftsforschung Münster, Westfälische Wilhelms-Universität Münster, Am Stadtgraben 9, 48143 Münster, Deutschland

E-Mail: gordon.klein@wiwi.uni-muenster.de 


\section{Public broadband subsidies - a skepical discussion}

Abstract The digitalization affects different aspects of the economy and the society. Not surprisingly, policy makers want to have an impact on this process. In particular different subsidy schemes are set up. In the following, some of them are described as an example. There is a particular focus on the subsidy scheme by the German federal government. At first, I describe the status-quo of the broadband rollout. Then, I provide an overview of the heterogeneous subsidy landscape. I evaluate whether there might be a normative scope for market interference. Finally, I discuss whether the current scheme is appropriate or not. I show that it may be too extensive and, thus, it may likely subsidize projects in those areas where there would have been a rollout - even without such a scheme - anyhow.

\section{Einleitung}

Die Digitalisierung ist seit Jahren eines der bestimmenden Themen in der wirtschaftspolitischen Diskussion. Dies gilt durch die jüngsten Corona Krise umso mehr, da z.B. ein vermehrter Einsatz von Home-Schooling und Home-Office sowie verstärkte Online-Bestellungen die steigende Relevanz digitaler Methoden und Infrastrukturen aufgezeigt hat.

Der Begriff der Digitalisierung ist sehr vielschichtig und beschreibt einen großen Prozess, eine Transformation, die viele Lebens- und Arbeitsbereiche umfasst. Kern und Auslöser dieser Entwicklung ist die Informations- und Kommunikationstechnologie (IKT). ${ }^{1}$ Diese fungiert als eine Querschnittstechnologie, oder auch als eine Basistechnologie, in der wirtschaftswissenschaftlichen Literatur auch „General Purpose Technologie“ genannt. Diese „General Purpose Technologien“ sind solche, die als Basistechnologie verschiedene andere Technologien ermöglichen. Der Begriff wurde von Bresnahan und Trajtenberg (1995) geprägt, die hierfür z.B. die Elektrizität oder die Dampfmaschine anfügen, deren Etablierung weitere komplementäre Technologien ermöglichten und eine große technologische und wirtschaftliche Entwicklung anschoben. Als eine solche Technologie wird auch die IKT angesehen (vgl. für einen Überblicksartikel zu den Auswirkungen von IKT vgl. auch Draca et al. 2007). Dies bedeutet, dass diese als Grundlagentechnologie viele andere Technologiebereiche und somit eine Transformation von Wirtschaft und Gesellschaft ermöglicht.

Chun et al. (2008) interpretieren den von IKT-induzierten Transformationsprozess, angelehnt an Schumpeter, als einen Prozess der kreativen Zerstörung. Dieser Begriff bedeutet, dass der Transformationsprozess alte Geschäftsmodelle zerstört und neue wiederum hervorbringt. Dies beschreibt somit etwas, was man im Alltag täglich beobachten kann. In traditionellen Branchen wie dem Einzelhandel, dem Zeitungsmarkt, oder als prägnantes Beispiel dem Videothekenmarkt wird dies sehr deutlich. Beim Blick in viele Innenstädte lässt sich ein gewisser Leerstand, auch durch Online-Shoppingkonkurrenz induziert, nicht verleugnen. Zudem sinken stetig

\footnotetext{
${ }^{1}$ Vgl. hierzu für ein analoges Argument, Bertschek et al. (2013, S. 190)
} 


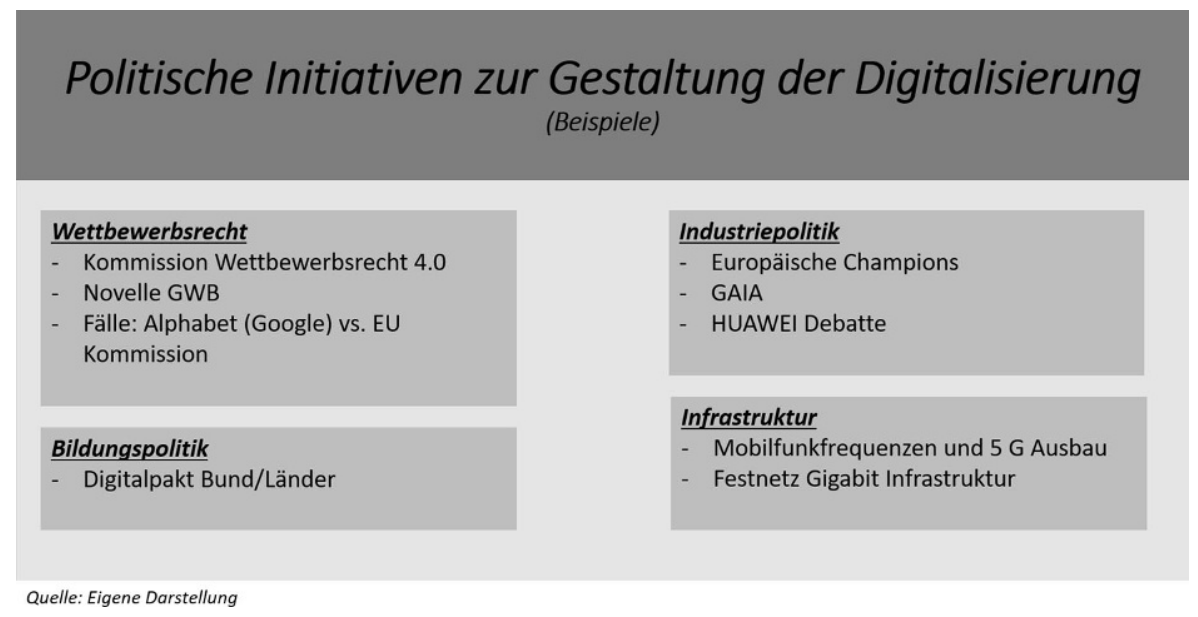

Abb. 1 Beispiele politischer Initiativen

die Zeitungsauflagen und Videotheken sind praktisch nicht mehr existent. Tatsächlich stehen diesem Niedergang eine Vielzahl an neunen Angeboten entgegen: OnlineHandel, Social Media oder Videostreaming.

Es liegt in der Natur der Sache, dass dieser Transformationsprozess von der Politik als herausfordernd angesehen wird. Folglich ist eine Vielzahl verschiedener politische Initiativen wenig überraschend. Beispiele hierfür sind in Abb. 1 aufgeführt.

Die dargestellten Beispiele bilden sehr unterschiedliche Initiativen ab, die hier beispielhaft in vier Bereiche aufgegliedert werden. So hat die Bundesregierung die Kommission Wettbewerbsrecht 4.0 einberufen um Notwendigkeiten und Ansätze der Umgestaltung des Wettbewerbsrechts im Hinblick auf Probleme der neuen digitalen Welt zu identifizieren (BMWi 2019). Auch wurde jüngst die 10. Novelle des GWB initiiert, die das Wettbewerbsrecht, gerade im Hinblick auf digitale Plattformmärkte und große Digitalfirmen insgesamt, neu justiert und dem Bundeskartellamt hierbei neue Spielräume für Eingriffe erlaubt (Gesetzentwurf zur 10. GWB-NOVELLE: BMWI 2020b). Große Aufmerksamkeit haben auf europäischer Ebene die Fälle der EU Kommission gegen den US-Konzern Alphabet, der insb. für seine Suchmaschine Google oder seine Videoplattform YouTube bekannt ist, erhalten. Hier wurden empfindliche Strafen für Marktmachtmissbrauch ausgesprochen (EU-Kommission 2019b).

Im Bereich der Industriepolitik fällt eine stete Diskussion für Europäische Champions auf, die sich nicht nur auf den Digitalbereich beschränkt (Tagesschau 2019). Als prominentestes Beispiel der Wirtschaftsförderung dürfte hier die Initiative GAIA sein, die darauf abzielt europäische Cloud-Lösungen, die unabhängig von außereuropäischer Jurisdiktion arbeiten und europäischen Datenschutz sicherstellen, zu ermöglichen (BMWi und BMBF 2019). Ebenso stellt sich im internationalen Kontext die Frage, wie mit dem Netzausrüster HUAWEI zu verfahren ist, der unter anderem sensible 5G-Ausrüstung anbietet und nun in mehreren westlichen Ländern vom Ausbau von 5G-Mobilfunknetzen ausgeschlossen wird (The Guardian 2020). 
Digitalisierung und Bildung sind insbesondere in der aktuellen Corona-Krise in den Fokus gerückt. Tatsächlich gab es durch den Digitalpakt und der Grundgesetzreform bereits große Ambitionen, die Digitalisierungsbestrebungen in den Schulen zu verstärken (BMWi 2020a). Nach Monaten des Home-Schoolings in der Corona-Krise hat sich die Debatte diesbezüglich noch deutlich verstärkt, da vielfältige Defizite aufgedeckt worden sind, die dann auch von der breiten Öffentlichkeit wahrgenommen wurden. Es ist daher damit zu rechnen, dass es in diesem Bereich noch deutlich mehr Bestrebungen geben wird.

Der letztgenannte Punkt in Abb. 1 betrifft die Infrastruktur. Während vor einigen Monaten die Debatte um die Versteigerung der 5G-Lizenzen, und verschiedener Ausbauverpflichtungen, im Fokus standen (Bundesnetzagentur 2019a), gibt es schon seit Jahren eine lebhafte Diskussion um den Ausbau von Breitbandinternet und insbesondere Fiber-to-the-Home (FTTH), also Glasfaseranschlüsse bis ins Haus. Letztlich ist die Forderung entstanden, dass gigabitschnelles Internet in allen Haushalten zugänglich sein sollte, wozu unter anderem FTTH als leistungsfähigste Infrastruktur in der Lage ist. Dass es eine Diskussion über Infrastruktur gibt, ist grundsätzlich wenig verwunderlich. Insbesondere gilt dies, da es ein ehemaliges staatliches Unternehmen gibt, das Subjekt von Zugangsregulierung ist. Die Frage der korrekten Regulierung unter der Abwägung von kurzfristigen Zielen des günstigen Zugangs mit den langfristigen Zielen des hoch-qualitativen Zugangs hat in der Vergangenheit, und auch aktuell, eine große Debatte in der Forschung befeuert (vgl. für einen Überblicksartikel Cambini und Jiang 2009). Tatsächlich geht es aber bei gigabitschnellen Netzen um neue Netzteile, für die der privatwirtschaftliche Ausbau als nicht hinreichend angesehen wird und daher ein differenziertes Förderregime aufgebaut worden ist (vgl. hierzu Abschn. 3).

Um diese Förderung soll es nun in dieser Arbeit gehen. Es wird dargestellt, dass die differenzierte und ambitionierte Förderung sich auf Bundesebene direkt von den Regierungszielen herleiten lässt und insgesamt durchaus großzügig ausgestaltet ist. Flankiert wird dies von Förderprogrammen der Länder. Bei einer solch großzügigen Förderung stellen sich mehrere Fragen, die hier behandelt werden. So gilt es zu fragen, ob eine Förderung grundsätzlich normativ zu rechtfertigen ist und dann, ob diese effizient, d.h. zielgerichtet, erfolgt. Diese Arbeit legt dar, dass erstere Frage grundsätzlich positiv zu beantworten ist, d.h., dass eine normative Rechtfertigung grundsätzlich vorliegt. Ebenso wird aufgezeigt, dass davon auszugehen ist, dass die Förderung mutmaßlich zu weit geht und dort fördert, wo dies im Grundsatz nicht unbedingt nötig ist. Stattdessen scheint es so, dass es weitere Probleme gibt, die einen Ausbau hemmen.

Die Arbeit ist wie folgt aufgebaut: Zuerst wird in Abschn. 2 der Stand des Breitbandausbaus in Deutschland aufgezeigt. Folgend wird in Abschn. 3 eine Übersicht über die Förderprogramme in Deutschland auf Bundes- und Landesebene dargestellt. Die Landesebene wird hierbei beispielhaft am größten Bundesland Nordrhein-Westfalen aufgezeigt. In Abschn. 4 erfolgt eine Diskussion normativer Rechtfertigungen einer Förderung und eines Markteingriffs, in Abschn. 5 erfolgt eine kritische Würdigung des Falls, in Abschn. 6 eine kurze Zusammenfassung und ein Fazit. 


\section{Stand des Breitbandausbaus in Deutschland}

Die EU-Kommission (2019a) evaluierte in einer Studie den Stand des Breitbandausbaus in Europa und zeigte dabei dezidiert auf, wie die Breitbandverfügbarkeit allgemein, und im Speziellen die Verfügbarkeit von sogenannten Next-GenerationAccess Networks (NGA, definiert als Geschwindigkeit größer 30 MBit/s), und solchen Netzen, die mehr als $100 \mathrm{Mbit} / \mathrm{s}^{2}$ ermöglichen, in den europäischen Ländern ausgeprägt ist.

Die Studie (EU-Kommission 2019a, S. 93 ff) legt dar, dass fast alle Haushalte in Deutschland, ebenso wie der europäische Durchschnitt, fast vollständig mit einfachem Breitband versorgt sind (99,9\%). ${ }^{3}$ Dabei haben 97,5\% einen Breitbandfestnetzanschluss (verglichen mit 96,7\% im EU-28-Durchschnitt). 87,9\% sind mit sogenannten Next-Generation Access Networks mit einer Geschwindigkeit von über $30 \mathrm{Mbit} / \mathrm{s}$ versorgt (verglichen mit 83,1\% im EU-28-Durchschnitt). Von Glasfaser bis zum Grundstück und DOCSIS-3.0-Technologie mit einer Geschwindigkeit von über $100 \mathrm{Mbit} / \mathrm{s}$ (perspektivisch schnell mit $1 \mathrm{GBIT} / \mathrm{s}$ ) sind 66,3\% (verglichen mit $59,8 \%$ im europäischen Durchschnitt) erschlossen. Insgesamt ist diese Verfügbarkeit im EU-28-Vergleich leicht überdurchschnittlich anzusehen. Vergleicht man die verschiedenen Festnetztechnologien, sieht man, dass Deutschland bei der DSL- (DE 97,4\% vs. EU-28: 92,2\%), VDSL- (DE 76,9\% vs. EU-28: 56,7\%) und Kabelinternetinfrastruktur (DOCSIS-3.0; DE 64,1\% vs. EU-28: 45,0\%) überdurchschnittlich ausgebaut ist, aber bei der leistungsfähigsten Infrastruktur, Glasfaser bis zum Grundstück (DE: 8,5\% vs. EU-28: 29,6\%), deutlich unter dem Durchschnitt versorgt ist.

Eine klare Bewertung ist hier schwierig. Hierbei ist auch zu berücksichtigen, dass die Werte für Deutschland als Ganzes angegeben sind. Wie auch in der Studie der Kommission angegeben, (EU-Kommission 2019, S. 93) sind die Werte im ländlichen Raum deutlich geringer (NGA: 66,2\%; DOCSIS-3.0 und Glasfaser zum Grundstück $18,0 \%$ ). Das bedeutet, dass unabhängig vom Gesamteindruck geografische Disparitäten problematisch sein könnten.

Mit Blick auf das Aggregat liegt Deutschland tatsächlich bei der Verfügbarkeit der typischen Geschwindigkeiten über dem Durchschnitt, aber nicht bei der leistungsfähigsten Infrastruktur. Ob dies ein tatsächliches Defizit ist, lässt sich nicht beurteilen. Insbesondere nicht dann, wenn man die Anschlussqualität technologieneutral und mit den im EU-Report angegebenen Geschwindigkeiten bewertet.

Trotzdem besteht bei der schnellsten Geschwindigkeitsstufe (Glasfaser bis zum Grundstück und DOCSIS-3.0) aktuell ein Ausbau von nur 65\%. Es besteht somit eine Diskrepanz von ca. 35\% der Haushalte, die aktuell noch nicht mit gigabitfähigen Internetanschlüssen ausgestattet sind (unter der Annahme eines vollständigen Ausbaus auf DOCSIS-3.1.). Hinzu kommt ein Ausbau der seit dem Beobachtungs-

\footnotetext{
${ }^{2}$ Die Angaben für solche Netze die mit mehr als 100 MBIT/s versorgt werden, sind solche, die mit Glasfaser bis zum Grundstück sowie Kabelinternet per DOCSIS-3.0-Standard erschlossen sind. Da durch den aktuellen Standard DOCSIS-3.1 diese Netze vergleichsweise einfach so ausgelegt werden können, dass Gigabit Geschwindigkeiten möglich sind, umfasst diese Kategorie somit Netze, die zumindest perspektivisch mit Geschwindigkeiten von $1 \mathrm{Gbit} / \mathrm{s}$ beschaltet werden können sollten (vgl. auch Monopolkommission 2019, S. 20, RZ. 19).

3 Die weiteren Werte dieses Abschnitts sind alle der Studie EU-Kommission (2019a) entnommen.
} 
zeitraum der Studie im Jahr 2019 mit dem Ausbaustand 2018 stattgefunden hat. D. h. es ist nicht gänzlich klar, ob bis zum heutigen Stand wesentliche Bereiche weiter ausgebaut worden sind.

\section{Förderung des Breitbandaus in Deutschland am Beispiel von NRW}

Der Ausbau von Breitbandinternetnetzen ist als übergeordnetes Ziel bereits im CDU/SPD Koalitionsvertrag von 2018 festgelegt: ,Wir gestalten den Weg in die Gigabit-Gesellschaft mit höchster Priorität. Deshalb wollen wir den flächendeckenden Ausbau mit Gigabit-Netzen bis 2025 erreichen. Wir wollen den Netzinfrastrukturwechsel zur Glasfaser. Unser Ziel lautet: Glasfaser in jeder Region und jeder Gemeinde, möglichst direkt bis zum Haus. Schulen, Gewerbegebiete, soziale Einrichtungen in der Trägerschaft der öffentlichen Hand und Krankenhäuser werden wir bereits in dieser Legislaturperiode direkt an das Glasfasernetz anbinden“ (CDU 2018). Hieraus leitet sich das Ziel der Bundesregierung, wie z. B. vom BMVI formuliert, ab: „Das übergeordnete Ziel ist superschnelles Internet mit mindestens 1 Gigabit/s in ganz Deutschland bis 2025. Schulen, Gewerbegebiete und Krankenhäuser werden prioritär in den Fokus der Förderung genommen.“" (BMVI 2020).

Aus diesem Ziel speisen sich wiederum verschiedene Bundesförderprogramme, die, von der Bundesregierung unabhängig und lokal durch Landesregierungen (hier natürlich von eigenen Zielen abgeleitet) flankiert werden. Abb. 2 stellt diese Programme mit dem lokalen Beispiel für NRW zusammen.

Das wesentliche Instrument des Bundes ist hier das Programm „Förderung zur Unterstützung des Breitbandausbaus in der Bundesrepublik Deutschland“, das eine Förderung für Breitbandausbauprojekte von 50\% (plus ein etwaiger Zuschlag

\section{Übersicht von Bundes und Länderförderprogrammen zum Gigabit-Breitbandausbau am Beispiel NRWs}

\begin{tabular}{|c|c|c|c|c|c|c|c|}
\hline \multicolumn{2}{|c|}{$\begin{array}{l}\text { Förderung zur Unterstützung } \\
\text { des Breitbandausbaus in der } \\
\text { Bundesrepublik Deutschland }\end{array}$} & \multicolumn{3}{|c|}{$\begin{array}{l}\text { Förderung von Infrastruktur- } \\
\text { projekten in Gewerbe- und } \\
\text { Industriegebieten sowie in } \\
\text { Häfen }\end{array}$} & \multicolumn{2}{|c|}{$\begin{array}{l}\text { Förderung von Infrastruktur- } \\
\text { projekten zur Anbindung von } \\
\text { Schulen und Krankhäusern }\end{array}$} & $\stackrel{\mathscr{D}}{\complement}$ \\
\hline $\begin{array}{l}\text { Kofinanzierung des } \\
\text { Bundesförderpro- } \\
\text { gramms }\end{array}$ & \multirow{2}{*}{\multicolumn{2}{|c|}{$\begin{array}{l}\text { Förderung von NGA- } \\
\text { Netzen im ländlichen } \\
\text { Raum in NRW }\end{array}$}} & \multirow[t]{2}{*}{$\begin{array}{l}\text { Förderung der } \\
\text { Breitbandversorgung } \\
\text { in ländlichen Räumen } \\
\text { in NRW (GAK) }\end{array}$} & \multirow{2}{*}{\multicolumn{2}{|c|}{$\begin{array}{l}\text { Regionales } \\
\text { Wirtschafts- } \\
\text { förderungsprogramm } \\
\text { (RWP) /GRW / EFRE }\end{array}$}} & \multirow{2}{*}{$\begin{array}{l}\text { Förderung der } \\
\text { Glasfaseranbindung } \\
\text { von öffentlichen } \\
\text { Schulen und } \\
\text { genehmigten } \\
\text { Ersatzschulen }\end{array}$} & \multirow[t]{2}{*}{$\begin{array}{l}\sum_{0} \\
\sum \\
\sum \\
\sum\end{array}$} \\
\hline & & & & & & & \\
\hline
\end{tabular}

Quelle: Eigene Darstellung in Anlehnung an Ministerium für Wirtschaft,

Innovation, Digitalisierung und Energie des Landes Nordrhein-Westfalen

Abb. 2 Übersicht Förderprogramme. (In Anlehnung an MfWIDuD des Landes NRW 2020) 
für Gebiete mit geringer Wirtschaftskraft) vorsieht. ${ }^{4}$ Die beiden anderen in Abb. 2 aufgeführten Programme zielen auf spezielle Projekte, d. h. Gewerbe- und Industriegebiete, Häfen, oder Schulen und Krankenhäuser, ab. Hier ist ebenso eine Förderung von $50 \%$ sowie etwaige Erhöhungen um $10 \%$ oder $20 \%$ vorgesehen, wobei die Fördermittel bei einer Million Euro gedeckelt sind und erst ab einer Projektgröße von $10.000 €$ starten. Des Weiteren sind spezifische Eigenanteile von $10 \%$ vorgegeben.

Wichtig ist hierbei, dass für die Gewährung einer Förderung ein Markterkundungsverfahren eingefordert wird. Die Monopolkommission (2019, S. 10, RZ K17) fasst dieses so zusammen, dass eine Umfrage bzgl. der Ausbaupläne der relevanten Unternehmen für die nächsten drei Jahre abgefragt werden. Nur wenn diese keine Pläne haben, kann der untersuchte Bereich gefördert werden. Die Monopolkommission führt an, dass grundsätzlich in der neusten Förderform alle Gebiete ohne Kabelinternet oder Glasfaseranschluss förderfähig sind. Dies Entspricht somit fast vollständig den ca. 30-35\%, die in Abschn. 2 als nicht mit der höchsten Geschwindigkeitskategorie versorgt, angegeben sind. Die Monopolkommission (2019, S. 10, RZ K17) weist darauf hin, dass gerade der im Markterkundungsverfahren abgefragte Zeithorizont von drei Jahren dazu führen kann, dass spätere privatwirtschaftliche Investitionen verdrängt werden könnten.

Die Landesprogramme kommen nun zusätzlich zu den dargestellten Bundesförderungsprogrammen hinzu. ${ }^{5}$ So gibt es eine explizite Beteiligung im Landesprogramm „Kofinanzierung des Bundesförderprogramms“, die den geförderten Betrag auf insgesamt $90 \%$ aufstockt. Darüber hinaus gibt es explizite Förderprogramme für NGA-Netze im ländlichen Raum, die $90 \%$ eines Wirtschaftlichkeitsfehlbetrags abdecken. Ebenso werden auch einfache Breitbandnetze im ländlichen Raum (mit bis zu 75\% des Wirtschaftlichkeitsfehlbetrags) oder Schulen und spezielle regionale Wirtschaftsförderprogramme gefördert.

Diese hier aufgeführten Programme zeigen klar auf, dass großer Aufwand betrieben wird, die in Abschn. 2 dargestellte Versorgungslücke, zu schließen. Da der Aufwand nicht nur in Höhe der Förderprogramme, sondern auch in Höhe der Förderung, einen nicht unerheblichen Aufwand hat (auf Bundesebene ca. eine Mrd. $€$ jährlich [vgl. Bundeshaushalt 2020; BMVI 2020]), stellt sich die Frage, ob eine Förderung grundsätzlich sinnvoll ist, ob die Förderung an den entscheidenden Ausbauhindernissen ansetzt, und ob diese trotz einer möglichen qualitativen Rechtfertigung in spezieller Höhe zu rechtfertigen ist.

\section{Normativ ökonomische Rechtfertigung für eine Förderung des Breitbandausbaus}

Für eine normative Betrachtung, ob eine Breitbandausbauförderung zum Schließen einer etwaigen Breitbandlücke vorliegt, muss zunächst geprüft werden, ob es ein

\footnotetext{
${ }^{4}$ Die Informationen der in diesem Abschnitt beschriebenen Programme sind von MfWIDuD (2020) entnommen.

${ }^{5}$ Ebenso wie im vorherigen Abschnitt wurden die Informationen in diesem Abschnitt von MfWIDuD (2020) entnommen.
} 
irgendwie geartetes Marktversagen auf dem Markt für Breitband oder auch Gigabitzugang gibt. D.h. es stellt sich zunächst die Frage, ob eine Nachfrage einzelner Individuen nicht durch private Anbieter bedient wird? D.h. haben die fehlenden 10-15\% der Haushalte für Breitbandanschlüsse, und etwa 30-40\% nach gigabitfähigen Breitbandanschlüssen, überhaupt eine nennenswerte Nachfrage nach solchen Anschlüssen (vgl. hierzu die Darstellung in Abschn. 2)? Diese Frage scheint nur auf den ersten Blick trivial. Betrachtet man die Internetnutzung in Deutschland, so zeigt sich, dass nicht die ganze Bevölkerung, sondern nur ca. $85 \%$ das Internet nutzen (Statista 2020). Zwar ließe diese Diskrepanz die Möglichkeit zu, dass diese eben nicht versorgten Haushalte überhaupt nicht an Breitbandinternetdiensten oder gigabitfähigen Internetdiensten interessiert sind. Da man jedoch davon ausgehen muss, dass sich diese Haushalte nicht gänzlich in ländlichen unterversorgten Gebieten isoliert von potenziell Nachfragenden bündeln, greift diese Logik nicht. Es ist davon auszugehen, dass es eine Vielzahl von potenziell Interessierten in den unterversorgten Gebieten gibt. Es ist jedoch anzunehmen, dass die absolute Zahl der Nicht-Versorgten eben auch solche beinhalten, die kein Interesse haben und somit die Verfügbarkeitszahlen das Problem potenziell überschätzen könnten.

Als Weiteres stellt sich die Frage, ob auf der Nachfrageseite nicht-internalisierte Faktoren bestehen, welche dazu führen, dass die individuelle Nachfrage kleiner als die optimale Nachfrage ist und somit zwar die soziale Nachfrage größer als die Kosten des Dienstes sind, nicht aber die individuellen Kosten, und es somit eine positive Externalität gibt. ${ }^{6}$

Es spricht viel dafür, dass es diese positiven Externalitäten grundsätzlich gibt. ${ }^{7}$ Die Literatur hat bereits in einer Vielzahl von Studien untersucht, ob Infrastrukturinvestitionen einen positiven Einfluss auf die wirtschaftliche Dynamik haben kann (vgl. für ein frühes Papier Aschauer 1989). So stellen Röller und Waverman (2001) in ihrem wegweisenden Artikel fest, dass öffentliche Investitionen in Telekommunikation einen kausalen positiven Einfluss auf wirtschaftliche Aktivität haben. Diese erhöhte Aktivität könnte man dann zum Teil als positive Externalität interpretieren. Diesem Klassiker haben dann weitere Forschungsarbeiten Evidenz für den Einfluss von Breitbandinternet auf die wirtschaftliche Aktivität hinzugefügt: So z. B., Koutroumpis (2009) und Czernich et al. (2011), deren Ergebnisse man in der selben Logik als positive Externalität des Breitbandinternets interpretieren kann.

D.h., es ist für Breitbandinternet insgesamt damit zu rechnen, dass dieses $\mathrm{zu}$ einer Steigerung der wirtschaftlichen Aktivität führt. ${ }^{8}$ Briglauer und Gugler (2019) finden ebenso einen positiven vergleichbaren Effekt von Glasfaserinternet, stellen diesen Effekt aber den Kosten gegenüber. Es ergeben sich in der Analyse Zweifel an dem kostenübersteigerten Ausbaunutzen für alle bisher unterversorgten Gebiete. An einem immer stetig steigenden Ausbaunutzen von mehr Geschwindigkeit erweckt auch die Studie von Ahlfeldt et al. (2017), für das Vereinigte Königreich, Zweifel.

\footnotetext{
${ }^{6}$ Wir gehen hier nur auf den Fall einer positiven Externalität, nicht hingegen auf den Fall einer negativen Externalität, ein.

7 Die folgende Diskussion der Literatur in diesem Absatz ist angelehnt an Klein (2020) und Bertschek et al. (2013).

${ }^{8}$ Die folgende Diskussion der Literatur in diesem Absatz ist angelehnt an Klein (2020).
} 
Sie untersuchen den individuellen Nutzen von Breitbandinternet, abgebildet durch den Einfluss auf Immobilienmärkte. ${ }^{9}$ Sie untersuchen hierbei explizit keine Glasfasertechnologie, finden aber für Breitbandinternet einen positiven Nutzen, allerdings mit abnehmenden Grenznutzen in der Geschwindigkeit. Dies bedeutet, dass der Nutzen immer schnellerer Technologien, also immer breitbandigerer Technologien, stets den Nutzen erhöht, dieser Nutzenanstieg jedoch mit steigender Geschwindigkeit sinkt. Dies lässt grundsätzlich Zweifel zu, ob jegliche Förderung, für immer schnellere Geschwindigkeit, stets zu rechtfertigen ist.

Die typische Art und Weise, wie man dieser Externalität begegnet, ist oftmals ein monetärer Ausgleich, der als Steuer für eine negative Externalität den Begriff „Pigousteuer“ innehat. ${ }^{10}$ Analog für eine positive Externalität wie hier, kann nun eine Förderung im Sinne einer Subvention erfolgen. Somit ist eine Förderung so wie sie eben auch stattfindet - eine grundsätzlich adäquate Art und Weise der Externalität zu begegnen. Im Gegensatz zu dem Grundmodell sei aber angemerkt, dass die Entscheidung eines Ausbaus eine binäre Entscheidung ist und eine Unternutzung hier nur darin besteht, dass es eben keine Infrastruktur gibt, die man nutzen könnte. Dies bedeutet, eine Förderung sollte in dem Sinne so ausgestaltet sein, dass nur solche Projekte realisiert werden sollen, bei denen privater und sozialer Nutzen größer als die Ausbaukosten sind. Wenn ein Projekt, d. h. ein Ausbau, bereits stattfindet, dann führt eine Subvention zu keiner Steigerung der Wohlfahrt. Stattdessen ist mit Schattenkosten der Mittelerhebung zu rechnen was einen Wohlfahrtsverlust zur Folge hat.

Über Externalitäten, die eben eine Förderung im Sinne einer negativen Pigousteuer rechtfertigen könnten, hinaus, sind alternative Gründe für Markteingriffe denkbar. Es könnte sein, dass es Gründe gibt, die dazu führen, dass es nicht zu einer Erreichung einer Minimalkostenkombination kommt, da der Anbieter nicht die Informationen erlangen kann, wer tatsächlich einen solchen Anschluss möchte, oder er von regulatorischen Gegebenheiten abgehalten wird, die Konsumenten zu bündeln, z. B. im Sinne einer Kundenbündelung. Einen Hinweis darauf, dass es in diesem Bereich grundsätzlich Diskussionen gibt, ist die jüngst umschlagende Haltung des Bundeskartellamts gegenüber des gemeinsamen Glasfaserausbaus des deutschen Marktführers und regulierten Incumbents, Deutsche Telekom, mit regionalen Firmen. Hier verbot das Bundeskartellamt in der Vergangenheit solche gemeinsamen Projekte, um jüngst eben solche, unter bestimmten Auflagen, zu erlauben (Bundeskartellamt 2019).

Weitere Möglichkeiten, die dazu führen könnten, dass eine existente Nachfrage nicht bedient wird, könnte die Marktstruktur und zu wenige aktive Infrastrukturwettbewerber sein. D.h., es könnte sein, dass die Deutsche Telekom, dadurch, dass sie zwar Wettbewerb im Servicemarkt hat (durch Regulierungsverpflichtungen für Vorleistungen), aber kaum Wettbewerb für Breitbandnetzanbieter, das Investitionsniveau zu gering ist. Als Hintergrund kann die von Aghion et al. (2005) dargebrachte Theorie, die zwischen Wettbewerb und Innovation eine umgekehrte U-Kurve postuliert, dienen. In der Analogie betrachtet, bedeutet dies, dass wenn die Telekom

9 In Anlehnung an die Diskussion in Klein (2020).

10 Vgl. für eine einfache Textbucheinführung Hindricks und Myles (2006, S. $188 \mathrm{ff})$. 
kaum Wettbewerber hat, ist der Anreiz gering in risikoreiche Ausbauprojekte zu investieren, da der Gewinn einer Investition beschränkt ist. Die Investition führte kaum zu mehr Marktanteilen und könnte nur eine höhere Zahlungsbereitschaft für höhere Qualität erzielen. Wenn von dieser quasi-monopolistischen Netzsituation nun aber zusätzlicher Wettbewerb aufkommt, kann eine Innovation nicht nur eine höhere Zahlungsbereitschaft hierdurch aufgreifen, sondern auch ein Abweichen der Kunden auf den Wettbewerber verringern. D.h. der Gewinn aus einer Investition steigt. ${ }^{11}$ Durch die Investition könnte neben einer erhöhten Zahlungsbereitschaft der bestehenden Kunden, zusätzlich neue Kunden generiert werden (bzw. weniger als in einer kontrafaktischen Situation verloren werden). Gemäß dieses Gedankengerüsts würde dann die steigende Relevanz von alternativen Anschlussbetreibern, insb. Kabelinternetbetreibern ${ }^{12}$, als großen überregionalen Netzwettbewerber, dazu führen, dass die Bestrebungen zu verstärkten Investitionen stiegen, da man von einer quasimonopolistischen Netzsituation zu einer Situation mit mehr Wettbewerb gelangt ist. Aus dieser Betrachtung kann z. B. die Fusion der großen Kabelinternetanbieter von Vodafone und Unitymedia (siehe zur Entscheidung der EU-Kommission zu dieser Fusion: EU-Kommission 2019c), als eine Rechtfertigung gesehen werden, hier aus Sicht der Wettbewerbsbehörden intermodalen Wettbewerb zu fördern.

In der Gesamtschau finden sich verschiedene normative Begründungen, Markteingriffe zu rechtfertigen. Die abgeleiteten Eingriffe sind hierbei jedoch sehr heterogen. Als tatsächliche Förderung durch eine Subvention lässt sich bei den hier diskutierten Gründen tatsächlich hauptsächlich eine Förderung durch zu erwartende Externalitäten sehen. D.h., es besteht die Frage, ob die individuelle Nachfrage ausreichend ist, solche Projekte zu finanzieren, oder eben nicht. Falls dies nicht der Fall sein sollte, ließe sich ein Eingriff rechtfertigen. Schlussendlich ist die Größe der Externalität schwierig zu bestimmen. Die benannten Studien geben hier Indizien. Wichtig ist es aber, dass die Zielgenauigkeit gesichert wird. D.h., nur solche Projekte gefördert werden, bei denen eben auch ein Externalitäten-Problem vorliegt und eben kein anderes der oben diskutierten Probleme.

\section{Kritische Diskussion des Bundesprogramms}

Wie bereits in Abschn. 3 dargelegt gibt es eine Vielzahl von Förderungen zum Breitband und insbesondere zum Gigabit-Ausbau in Deutschland. Dabei gibt es, wie dargestellt, verschiedene Bundesprogramme, die von unterschiedlichen Landesprogrammen flankiert werden. Dabei wird, wie am Beispiel von NRW gezeigt, teilweise

\footnotetext{
11 Des Weiteren postuliert diese Theorie, dass im atomistischen Wettbewerb der Anreiz zur Investition ebenso gering ist. D.h. eine gewisse Marktkonzentration mit aktiven Wettbewerb ist das Innovations-, bzw., hier in der Analogie, investitionsfreundlichste Umfeld.

12 Von 2009-2018 ist die Anzahl alternativer Technologien und insb. von Kabelinternet von 2,6 Mio. Anschlüsse auf 9,4 Mio. Anschlüssen gestiegen, wobei klassische DSL Dienste von 22,4 auf 25,2 Mio. Anschlüsse gestiegen. vgl. Bundesnetzagentur (2019b, S. 25, Abb. 4). Da klassische DSL-Dienste typischerweise Vorleistungsprodukte der Deutschen Telekom beinhalten und diese den Hausanschluss typischerweise besitzt (vgl. Bundesnetzagentur 2019b, S. 27), sprechen Zuwächse in alternative Infrastruktur für wachsenden Infrastrukturwettbewerb.
} 
die Förderung des Bundes noch aufgestockt. Wie bereits am Anfang dargestellt, ist das Bundesprogramm das größte und somit für eine Analyse das Interessanteste.

Dabei sieht das Bundesprogramm eine Förderung von 50\% des Projektvolumens vor (vgl. auch Abschn. 3). Dies bedeutet allerdings nicht, dass jeglicher Ausbau gefördert wird, sondern, dass dies weiter konditionalisiert wird. Insbesondere muss ein sogenanntes Markterkundungsverfahren durchgeführt werden (vgl. BMVI 2020), bei dem geprüft wird, ob es privatwirtschaftliches Interesse für einen Netzausbau gibt (innerhalb der nächsten drei Jahre). Wie die Monopolkommission (2019, Rz $149 \mathrm{ff}$ ) darstellt, sollen die Bedingungen des Verfahrens so angepasst werden, dass nicht nur unversorgte Bereiche gefördert werden, sondern auch solche, bei denen bereits eine teilweise breitbandige Infrastruktur vorhanden ist. Somit wird fast das gesamte Drittel, für das keine gigabitfähige Infrastruktur vorhanden ist, (vgl. Abschn. 2) förderfähig (vgl. Monopolkommission 2019, Rz. 149ff). Hierdurch erwartet die Kommission ein Verdrängen privater Investitionen, da zumindest in Teilgebieten ein Ausbau privatwirtschaftlich möglich wäre. Das geplante Markterkundungsverfahren wird hier als nicht ausreichend für eine effektive Verhinderung einer Verdrängung angesehen. ${ }^{13}$

Die Sicht, dass das Förderprogramm Gefahr läuft eine Überförderung darzustellen, wird von der jüngsten Studie von Klein (2020) unterstützt. So analysiert Klein (2020) wie der individuelle Nutzen eines Glasfaserausbaus zu bemessen ist. Ähnlich zu Ahlfeldt (2017) wird dazu der Einfluss von Breitbandinternet, hier aber direkt von Glasfaserinternet, auf Immobilienpreise bestimmt. Datengrundlage liefert hier der Ausbau in einem ländlichen Kreis, dem Kreis Warendorf. Das Differenzen-inDifferenzen-Setting kann hierbei einen Einfluss von gut $2 \%$ auf die Mietpreise darstellen. Dies bedeutet, dass es pro $100.000 €$ Immobilienwert $2000 €$ Wertzuwachs gibt. D. h. es werden signifikante Wertzuwächse, die sich auch in der Zahlungsbereitschaft widerspiegeln sollten, generiert. Klein (2020) vergleicht diese Wertzuwächse dann mit durchschnittlichen Projektkosten pro Anschluss, die aus dem Bericht der Monopolkommission (2019, S. 56) entnommen werden. Diese Projektkosten pro Anschluss entsprechen ungefähr $4400 €$ pro geförderten Projekt. Es ist klar, dass bei Immobilien mit einem Wert von 200.000-300.000€ dieser Wert regelmäßig durch den Wertzuwachs überschritten sein sollte. Unter der Annahme, dass der Kreis Warendorf ein typischer ländlicher Kreis sein sollte, ergibt sich die Schlussfolgerung, dass es in den grauen Bereichen, d.h. den Bereichen, in denen es breitbandigen Ausbau gibt, der allerdings nicht ein gigabitschnelles Netz umfasst, nicht die Regel sein sollte, dass der individuelle Nutzen eines Anschlusses nicht getragen wird. Es ist somit zu befürchten, dass die von der Monopolkommission (2019, Rz. $149 \mathrm{ff}$ ) vorgebrachte Befürchtung zu einem großen Teil eintreten kann.

Wenn es aber nun ein ca. 30\% der Haushalte umfassendes Gefälle bei der Versorgung mit gigabitfähigen Breitbandanschlüssen gibt, obwohl eine objektivierbare Zahlungsbereitschaft ausreichen sollte, stellt sich die Frage, wieso der Ausbau nicht

\footnotetext{
13 Das Verdrängen privatwirtschaftlicher durch geförderte Projekte lässt sich auch, wie in Abschn. 4 dargestellt vereinfacht deshalb als ein Problem sehen, wenn man unterstellt, dass ein Projekt auch ohne Subvention stattfände, aber das Generieren von öffentlichen Mitteln Schattenkosten (Kosten und Ineffizienzen durch Steuererhebung) beinhaltet.
} 
großflächiger stattfindet. Hierbei können die in Abschn. 4 weiter diskutierten Gründe zählen. D.h., regulatorische Hemmnisse zur Kundenbündelung oder marktstrukturbedingte Hemmnisse könnten vorliegen. Zum Beispiel kann es sein, dass Kooperationen nicht gestattet werden. Auch könnten Marktstrukturprobleme Hemmnisse darstellen oder Veränderungen wie die Fusion Unitymedia/Vodafone, noch positive oder negative Effekte über den Zeitablauf haben.

Letztlich kann auch das regulatorische Framework zur Netzregulierung Hemmnisse enthalten. Es ergibt sich ein weites Feld für etwaige Bedarfe wirtschaftspolitischer Eingriffe und/oder regulatorischer Anpassungen. Auch können einfach zeitliche Diskrepanzen vorliegen, d.h. die objektivierbare Zahlungsbereitschaft ist theoretisch abzuleiten, aber einigen potenziellen Kunden im Einzelfall noch nicht bewusst, bzw. die Zahlungsbereitschaft könnte über die Zeit noch steigen. Auch ist es denkbar, dass Tiefbaukapazitäten nicht unbegrenzt vorliegen und es somit eine Rangfolge für Investitionen von sehr lohnenden in weniger, aber immer noch lohnenden Gegenden gibt (vgl. hierzu auch Monopolkommission 2019, S. 10-12).

\section{Zusammenfassung und Diskussion}

Diese Arbeit hat aufgezeigt, dass verschiedene Aspekte der Digitalisierung aktuell diskutiert werden und beschäftigt sich insbesondere mit der Infrastrukturseite, d.h. dem Ausbau von Breitbandinternet. Hierbei steht gigabitschnelles Internet im Fokus.

Eine Betrachtung des Breitbandausbaus in Deutschland hat aufgezeigt, dass gigabitschnelles Breitbandinternet für einen maßgelblichen Teil, ca. einem Drittel der Anschlüsse, aktuell nicht zur Verfügung steht.

Dieser Zustand wird von der Politik als Problembereich angesehen. D.h., es ist das erklärte Ziel der Bundesregierung, dass auch diese Haushalte, die aktuell kein gigabitschnelles Breitbandinternet zur Verfügung haben, einen solchen Anschluss erhalten. Eine Betrachtung von normativen Begründungen einer möglichen Förderung hat hierbei aufgezeigt, dass insbesondere Externalitäten eine direkte Förderung rechtfertigen könnten.

Die Analyse der Literatur zeigt zum einen auf, dass es Grund zur Vermutung von wesentlichen positiven Externalitäten gibt, so dass eine Förderung grundsätzlich in Betracht kommen könnte. Im Hinblick auf eine solche Förderung, die nur dort eingreifend wirkt, wo es eine tatsächliche Lücke zwischen der individuellen Zahlungsbereitschaft und den Kosten des Ausbaus gibt, scheint es jedoch so zu sein, dass die Förderung zu weitgehend und undifferenziert auch solche Bereiche einschließt, die eben nicht gefördert werden müssten.

Es ist hierbei die Frage aufgeworfen worden, ob es, bei individuellem Nutzen, der größer als die Ausbaukosten des Glasfaserinternets ist, alternative Erklärungen für eine mutmaßliche Ausbaulücke geben könnte. Obgleich eine Detailbetrachtung hier nicht vorgenommen wurde, und den Rahmen dieser Arbeit sprengt, wurden mangelnder Infrastrukturwettbewerb oder zeitliche Friktionen, d. h. begrenzte Ausbaukapazitäten und sequentieller Ausbau, angedacht.

Als Fazit folgt daher, dass tatsächliche Ausbauförderung möglichst zielgenau dort angebracht ist, wo eine Diskrepanz zwischen Kosten des Ausbaus und des indivi- 
duellen Nutzens (nicht des sozialen Nutzens) der Konsumenten besteht. Darüber hinaus sollte eine differenzierte Analyse möglicher Ausbauhemmnisse erfolgen, um Instrumente zielgenauer einzusetzen.

Danksagung Ich bedanke mich für hilfreiche Kommentare der Mitglieder des Ausschusses für Wirtschaftspolitik des Vereins für Socialpolitik, 2020 in Berlin sowie für Anmerkungen von Jan Kaumann, Samet Kibar, Aleksandra Komineck, Gregor Rippel und Matthias Vogt.

Funding Open Access funding enabled and organized by Projekt DEAL.

Open Access Dieser Artikel wird unter der Creative Commons Namensnennung 4.0 International Lizenz veröffentlicht, welche die Nutzung, Vervielfältigung, Bearbeitung, Verbreitung und Wiedergabe in jeglichem Medium und Format erlaubt, sofern Sie den/die ursprünglichen Autor(en) und die Quelle ordnungsgemäß nennen, einen Link zur Creative Commons Lizenz beifügen und angeben, ob Änderungen vorgenommen wurden.

Die in diesem Artikel enthaltenen Bilder und sonstiges Drittmaterial unterliegen ebenfalls der genannten Creative Commons Lizenz, sofern sich aus der Abbildungslegende nichts anderes ergibt. Sofern das betreffende Material nicht unter der genannten Creative Commons Lizenz steht und die betreffende Handlung nicht nach gesetzlichen Vorschriften erlaubt ist, ist für die oben aufgeführten Weiterverwendungen des Materials die Einwilligung des jeweiligen Rechteinhabers einzuholen.

Weitere Details zur Lizenz entnehmen Sie bitte der Lizenzinformation auf http://creativecommons.org/ licenses/by/4.0/deed.de.

\section{Literatur}

Aghion, P., Bloom, N., Blundell, R., Griffith, R., \& Howitt, P. (2005). Competition and innovation: an inverted-U relationship. Quarterly Journal of Economics, 120(2), 701-728.

Ahlfeldt, G., Koutroumpis, P., \& Valletti, T. (2017). Speed 2.0: valuing universal access to digital highways. Journal of the European Economic Association, 15(3), 586-625.

Aschauer, D. (1989). Is public expenditure productive? Journal of Monetary Economics, 23(2), 177-200.

Bertschek, I., D. Cerquera, \& G.J. Klein. 2013. More Bits - More Bucks? Measuring the Impact of Broadband Internet on Firm Performance. Information Economics and Policy, 25(3):190-203.

BMVi (2020). Von der digitalen in die Gigabit-Gesellschaft. https://www.bmvi.de/SharedDocs/DE/ Dossier/Breitbandausbau/breitbandausbau.html. Zugegriffen: 6. Okt. 2020.

BMWi (2019). Ein neuer Wettbewerbsrahmen für die Digitalwirtschaft - Bericht der Kommission Wettbewerbsrecht 4.0. https://www.bmwi.de/Redaktion/DE/Publikationen/Wirtschaft/bericht-derkommission-wettbewerbsrecht-4-0.pdf?_blob=publicationFile\&v=12. Zugegriffen: 13. Okt. 2020.

BMWi (2020a). Digitalpakt Schule. https://www.digitalpaktschule.de/de/was-ist-der-digitalpakt-schule1701.html. Zugegriffen: 12. Okt. 2020.

BMWi (2020b): Entwurf eines Gesetzes zur Änderung des Gesetzes gegen Wettbewerbsbeschränkungen für ein fokussiertes, proaktives und digitales Wettbewerbsrecht 4.0 und anderer wettbewerbsrechtlicher Bestimmungen (GWB-Digitalisierungsgesetz) - Gesetzentwurf der Bundesregierung. https:// www.bmwi.de/Redaktion/DE/Downloads/Gesetz/gesetzentwurf-gwb-digitalisierungsgesetz.pdf? blob=publicationFile\&v=6 . Zugegriffen: 13. Okt. 2020.

BMWi, \& BMBF (2019). Das Projekt GAIA-X. https://www.bmwi.de/Redaktion/DE/Publikationen/ Digitale-Welt/das-projekt-gaia-X.pdf?_blob=publicationFile\&v=18. Zugegriffen: 12. Okt. 2020.

Bresnahan, T.F., \& Trajtenberg, M. (1995). General purpose technologies 'Engines of growth'? Journal of Econometrics, 65(1), 83-108.

Briglauer, W., \& Gugler, K.P. (2019). Go for gigabit? First evidence on economic benefits of (ultra-)fast broadband technologies in Europe. The Journal of Common Market Studies, 57(5), 1071-1090.

Bundeshaushalt (2020). Bundeshaushalt 2020 Einzelposten. https://www.bundeshaushalt.de/\#/2020/soll/ ausgaben/einzelplan/1204.html. Zugegriffen: 12. Okt. 2020.

Bundeskartellamt (2019). Telekom und EWE dürfen zum gemeinsamen Glasfaserausbau Gemeinschaftsunternehmen gründen [Pressemiteilung. https://www.bundeskartellamt.de/SharedDocs/Meldung/DE/ 
Pressemitteilungen/2019/30_12_2019_DTAG-EWE.html\#: :text=Telekom\%20und\%20EWE\%20d

$\%$ C3\%BCrfen\%20zum\%20gemeinsamen\%20Glasfaserausbau\%20Gemeinschaftsunternehmen $\% 20$ gr\%C3\%BCnden,-Meldung\%20vom\%3A\%2030.12\&text=Das\%20Bundeskartellamt\%20hat \%20heute\%20das,im\%20Rahmen\%20der\%20Fusionskontrolle\%20freigegeben. Zugegriffen: 13. Okt. 2020.

Bundesnetzagentur (2019a). Frequenzversteigerung beendet, Pressemitteilung. https://www.bundesnetz agentur.de/SharedDocs/Pressemitteilungen/DE/2019/20190612_Frequenzauktion.html. Zugegriffen: 13. Okt. 2020.

Bundesnetzagentur (2019b). Tätigkeitsbericht - Telekomunikation 2018/2019. https://www.bundesnetz agentur.de/SharedDocs/Mediathek/Taetigkeitsberichte/2019/TK_20182019.pdf? blob=publicationFile\&v=9. Zugegriffen: 13. Okt. 2020.

Cambini, C., \& Jiang, Y. (2009). Broadband investment and regulation: a literature review. Telecommunications Policy, 33(10-11), 559-574.

CDU (2018). Ein neuer Aufbruch für Europa Eine neue Dynamik für Deutschland Ein neuer Zusammenhalt für unser Land - Koalitionsvertrag zwischen CDU, CSU und SPD - 19. Legislaturperiode, Zeile: 1626-1632. https://www.cdu.de/system/tdf/media/dokumente/koalitionsvertrag_2018.pdf?file=1. Zugegriffen: 13. Okt. 2020.

Chun, H., Kim, J. W., Morck, R., \& Yeung, B. (2008). Creative destruction and firm-specific performance heterogeneity. Journal of Financial Economics, 89(1), 109-135.

Czernich, N., Falck, O., Kretschmer, T., \& Woessmann, L. (2011). Broadband infrastructure and economic growth. The Economic Journal, 121(552), 505-532.

Draca, M., Sadun, R., \& Van Reenen, J. (2007). Productivity and ICTs: a review of the evidence, the oxford handbook of information and communication technologies. New York: Oxford University Press.

EU-Kommission (2019a). Broadband coverage in Europe 2018-mapping progress towards the coverage objectives of the digital agenda. https://op.europa.eu/en/publication-detail/-/publication/81f5e7134ed8-11ea-aece-01aa75ed71a1. Zugegriffen: 13. Okt. 2020.

EU-Kommission (2019b). Milliardenstrafe gegen Google. https://ec.europa.eu/germany/news/20190320milliardenstrafe-google_de. Zugegriffen: 12. Okt. 2020.

EU-Kommission (2019c). Vodafone kann das Kabelgeschäft von Liberty Global unter Auflagen übernehmen, Pressemitteilung. https://ec.europa.eu/germany/news/20190718-vodafone-liberty-global_de. Zugegriffen: 12. Okt. 2020.

Hindricks, J., \& Gareth, M.D. (2006). Intermediate public economics (1. Aufl.). Cambridge, Massachussets: MIT Press.

Klein, G. (2020). Fiber-Broadband-Intenet and Its Regional Impact-An Empirical Investigation. CAWM Discussion Paper.

Koutroumpis, P. (2009). The economic impact of broadband on growth: a simultaneous approach. Telecommunications Policy, 33(9), 471-485.

MfWIDuD (2020). „Übersicht Förderprogramme“ des Kompetenzzentrums GIGABIT.NRW, Ministerium f Wirtschaft, Innovation, Digitalisierung und Energie des Landes NRW. https://www.gigabit.nrw. de/images/PDFs/Foerderrichtlinien/1908027_Tabelle_Foerderprogramme.pdf. Zugegriffen: 12. Okt. 2020 .

Monopolkommission (2019). 11. Sektorgutachten Telekommunikation. https://monopolkommission.de/ images/PDF/SG/11sg_telekommunikation.pdf. Zugegriffen: 12. Okt. 2020.

Röller, L.-H., \& Waverman, L. (2001). Telecommunications infrastructure and economic development: a simultaneous approach. The American Economic Review, 9(7), 909-923.

Statista (2020). Internetnutzung in Deutschland - Statista Dossier. https://de.statista.com/statistik/studie/ $\mathrm{id} / 22540 /$ dokument/internetnutzung-in-deutschland-statista-dossier/. Zugegriffen: 13. Okt. 2020.

Tagesschau (2019). EU-Kommission untersagt Zugfusion. https://www.tagesschau.de/wirtschaft/siemensalstom-115.html. Zugegriffen: 12. Okt. 2020.

The Guardian (2020). Europe divided on Huawei as US pressure to drop company grows. https:// www.theguardian.com/technology/2020/jul/13/europe-divided-on-huawei-as-us-pressure-to-dropcompany-grows. Zugegriffen: 12. Okt. 2020. 